\title{
Radiation damage in biological material: electronic properties and electron impact ionization in urea
}

\author{
C. Caleman ${ }^{1}$, C. Ortiz ${ }^{2}$, E. Marklund ${ }^{3}$, F. Bultmark ${ }^{2}$, M. Gabrysch ${ }^{4}$, F. G. Parak ${ }^{1}$, J. Hajdu ${ }^{3}$, M.

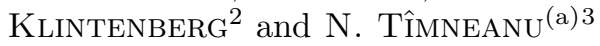

1 Physik Department E17, Technische Universität München - James-Franck-Strasse, DE-85748 Garching, Germany 2 Department of Physics and Material Science, Uppsala University - Angströmlaboratoriet, Box 530, SE-75121 Uppsala, Sweden

3 Department of Cell and Molecular Biology, Uppsala University - Biomedical Centre, Box 596, SE-75124 Uppsala, Sweden

${ }^{4}$ Department of Engineering Sciences, Uppsala University - Angströmlaboratoriet, Box 534, SE-75121 Uppsala, Sweden

$\begin{aligned} \text { PACS } 87.15 . \mathrm{A}- & \text { Biomolecules: structure and physical properties - Theory, modeling, and com- } \\ & \text { puter simulation } \\ \text { PACS } 71.20 . \mathrm{Rv}- & \text { Electron density of states and band structure of crystalline solids - Polymers } \\ & \text { and organic compounds } \\ \text { PACS } 79.20 . \mathrm{Hx}- & \text { Electron and ion emission by liquids and solids; impact phenomena - Electron } \\ & \text { impact: secondary emission }\end{aligned}$

\begin{abstract}
Radiation damage is an unavoidable process when performing structural investigations of biological macromolecules with X-rays. In crystallography this process can be limited through damage distribution in a crystal, while for single molecular imaging it can be outrun by employing short intense pulses. Secondary electron generation is crucial during damage formation and we present a study of urea, as model for biomaterial. From first principles we calculate the band structure and energy loss function, and subsequently the inelastic electron cross section in urea. Using Molecular Dynamics simulations, we quantify the damage and study the magnitude and spatial extent of the electron cloud coming from an incident electron, as well as the dependence with initial energy.
\end{abstract}

Introduction. - Recent advances in the development of X-ray Free Electron Lasers (XFEL) offer a tantalizing ability to do photon science using short intense X-ray pulses. As a consequence, a number of theoretical models describing the physics of extreme X-ray-material interaction have been presented [1-6]. The potential to use the XFEL to do 3D single bioparticle imaging [1] has enhanced the efforts to theoretically describe the dynamics of a sample being exposed to an XFEL pulse. On a time scale longer than $5 \mathrm{fs}$, most of the ionizations in a sample exposed to an X-ray pulse will not be due to primary photo ionizations, but due to inelastic electron scattering. In the aftermath of a single photo ionization, the photo electron as well as consecutive Auger electrons will interact with outer shell electrons in the surrounding atoms, leading to an electron cascade (electron shower). This process

\footnotetext{
(a) corresponing author, email: nicusor@xray.bmc.uu.se
}

is exploited when detecting photons in photo-multipliers, but leads to undesired ionization and structure damage in diffractive imaging. It is therefore important to know the inelastic scattering properties and understand the dynamics of the electron generation when describing the damage process. The inelastic cross section provides information regarding the deposition of energy from an energetic electron into the system. For free atoms and ions the electron cross sections are known, and there are a few simple empirical expression presented in the literature that give good estimation of the free atomic cross sections, see for example ref. [7].

Structural determination of biological relevant samples, such as proteins, viruses or cells, are of large interest in many branches of life science. In the near future, facilities such as the European XFEL source in Hamburg and the LINAC Coherent Light Source (LCLS) at the Stanford 
Linear Accelerator Center, as well as table-top XFEL [8] have promised to provide short hard X-ray pulses, at wavelengths suitable for atomic or near-atomic structural determination of biological molecules. To describe the secondary electron production in biomolecular samples, we present a theoretical study of urea, in which the cross sections were derived from first principle calculations, using a methodology developed and tested earlier $[9,10]$.

Method. - We perform simulations of the electron cascade in crystalline urea $\left(\mathrm{CO}\left(\mathrm{NH}_{2}\right)_{2}\right)$ using the spatial electron dynamics program, EHOLE, that is a part of the Gromacs [11] Molecular Dynamics software package. To do these simulations we proceed according to the following work flow: (i) calculate the band structure and energy loss function (ELF), (ii) calculate the elastic and inelastic electron cross sections using the energy loss function for the latter, and finally (iii) simulate the electron cascade from a single electron at a certain energy.

Band structure and energy loss function from first principle calculations. The method used to calculate the inelastic cross section is based on the ability to calculate the energy loss function from first principle calculations. Performing such calculations requires a sample with a relatively few atoms in the unit cell. We have chosen to use urea as model for a biological sample due to three major reasons. Urea has a well known crystalline structure [12]. Urea has an atomic composition of biological character, containing light elements such as carbon, nitrogen, oxygen and hydrogen, and having a density close to the density of cellular constituents. Furthermore, the urea crystal is among the simplest crystalline organic materials known, with 16 atoms per unit cell in the tetragonal space group $P \overline{4} 2{ }_{1} m$.

The band structure of an idealized urea crystal is determined from first principles using structural data found in the literature $[13,14]$. We use the ABINIT first principle computer code [15] to generate electronic structure results within the Local Density Approximation (LDA). In our calculations, we use a $12 \times 12 \times 12$ reciprocal space grid and a plane wave cutoff value of $680 \mathrm{eV}$ for standard LDA pseudo-potentials. Additionally, a scissors operator of $1.46 \mathrm{eV}$ is used to correct the underestimation of the LDA band gap to optical data [16].

Electron cross sections. The generation of inelastic electron cross sections from energy loss functions is well known, and several methods are found in the literature. The method we use here is the so called TanumaPowell-Penn model (TPP), based on Bethe [17] and Lindhard's [18] work. We refer the reader to references [19-22] for a detailed information about the model. In earlier publications we have, in addition to TPP, employed an alternative model developed by Ashley et al. [23-25]. In the present work we chose to use only the TPP approach, since Ashley model was found to underestimate the number of generated electrons [26]. The approach of using calculated energy loss functions when determining the inelastic cross sections and secondary electron cascades there from, has been presented and evaluated in the case of two semiconducting materials in an earlier work [10]. To our knowledge, experimentally determined electron loss functions for urea are not available and we are in this work obliged to rely on an ELF calculated from first principles for the generation of the electron cross sections. Estimations of the inelastic electron cross sections have been presented earlier, both based on calculated and experimentally derived optical constants for biological material [27], as well as direct fitting to inelastic experimental data [28-30]. Pioneering work in determining the energy loss function in biomaterial from first principle calculations was initiated by Zaider and coworkers [31]. Tanuma et al. [28] present a study of the electron mean free paths between 50 and 2000 $\mathrm{eV}$ for 14 organic compounds, comparing and evaluating two fittings to the so called TPP approach (employed in the present work).

The elastic cross section is calculated with the Barbieri/van Hove Phase Shift package using a partial wave expansion technique $[32,33]$. The elastic cross section has limited impact on the final characteristics of the generated cascades. Further details on these calculations are presented in [9, 26, 34].

Electron generation and dynamics. Once we have determined the electron cross sections, we simulate the electron cascade as follows: (i) an initial electron with a certain energy is generated. For each time step we calculate the probability for inelastic or elastic collisions from the cross sections. The probability for any collision event is compared to a random number which determines whether the electron is scattered or not. (ii) When the electron scatters inelastically the energy loss of the electron is possible through two channels: (a) if the energy loss is higher than the binding energy of the outer shell electron ( $\left.E_{\text {lost }}>E_{\text {bind }}\right)$, an electron carrying a kinetic energy equal to the energy lost by the first electron minus the binding energy $\left(E_{\text {kin }}=E_{\text {lost }}-E_{\text {bind }}\right)$ is released. Now the system contains two electrons with the energy of the initial electron minus the binding energy of the second electron. These two electrons are now separately treated as the initial electron was. The cascades evolve independently for each electron. (b) If the energy loss by the initial electron is less than the binding energy $\left(E_{\text {lost }}<E_{\text {bind }}\right)$, the initial electron changes its path and loses the energy to the system. (iii) In the case of an elastic scattering event, the initial electron keeps its kinetic energy and changes its path, according to the elastic differential cross section. The generation of new electrons through impact ionization carries on until all electrons have a kinetic energy lower than the binding energy. Each electron trajectory is explicitly followed, and the kinetic energy and momentum of each individual electron is known at every time step. A time step of one attosecond was used in the simulations.

For computational simplicity we have assumed that all energy losses in the scattering events are absorbed 
by the system. The model does not include hole scattering, electron-hole recombinations, nor electron-phonon coupling. Neglecting the scattering of the holes accounts for a lowering of the total number of the ionizations events. In diamond this has been shown to underestimate the total number of electrons generated nearly by a factor of two compared to experiments [26,35]. Previous results for water, however, have shown good agreement with radiolysis data from experiments, albeit the measurements were performed on longer time scales [36].

Electron-hole recombination - where electrons and holes recombine and deposit energy into the system as phonons, photons, or excitations - is in principle part of the scattering dynamics. However, the recombination coefficient for urea was not found in the literature, and since the coefficients for other organic semiconducors span many orders of magnitude (see e.g. refs. [37-39]), estimates from analogous compounds will most likely be gravely misleading. We have chosen to ignore recombinations in our calculations, knowing it could potentially overestimate the number of ionizations, as electrons that would recombine cannot generate more electrons. However, the impact on the results will be limited as long as the carrier density is moderate, which is the case for all the electron cascades generated in this study.

In an earlier experimental study we have followed the formation of secondary electron cascades in single-crystalline diamond caused by femtosecond X-ray pulses [35]. The experimental results showed good agreement with a theoretical calculation based on the TPP model [26], when excluding electron-hole recombination. Furthermore, as shown in the next section, the large band gap calculated for urea $4.74 \mathrm{eV}$ is close to that of diamond $5.46 \mathrm{eV}$. Since the density of crystalline urea is lower than that of diamond $\left(\rho_{\text {diamond }} \approx 3.5 \mathrm{~g} / \mathrm{cm}^{3}, \rho_{\text {urea }} \approx 1.37\right.$ $\mathrm{g} / \mathrm{cm}^{3}$ ), we expect the electron-hole recombination in urea to be lower than in diamond.

Estimations of the vibrational spectra and the electronphonon coupling strength of the urea crystal require an accurate evaluation of the forces acting on the molecule upon intermolecular displacements. In its solid phase, urea has a planar configuration with vibrational modes ranging between $2.5 \mathrm{meV}$ to $439 \mathrm{meV}$ at $10 \mathrm{~K} \mathrm{[40].} \mathrm{Hence,} \mathrm{the} \mathrm{en-}$ ergy losses are expected to be somewhat larger than for diamond [26], but neglected here.

Dissociative Electron Attachment (DEA), i.e. fragmentation following electron capture at subionization energies, has been demonstrated to play an important role in radiation damage of biomolecular samples $[41,42]$. No experimental DEA cross sections for urea are known, and although cross sections for several biomolecules can be found in literature, the majority are for the gas phase. Experimental DEA cross sections for tetrahydrofuran [43] - a rudimentary analogue to the deoxyribose rings in DNA and acetone [44], both in the condensed phase, are nearly zero below 6 and $8 \mathrm{eV}$, respectively, and have peaks that are almost two orders of magnitude less than our calcu-

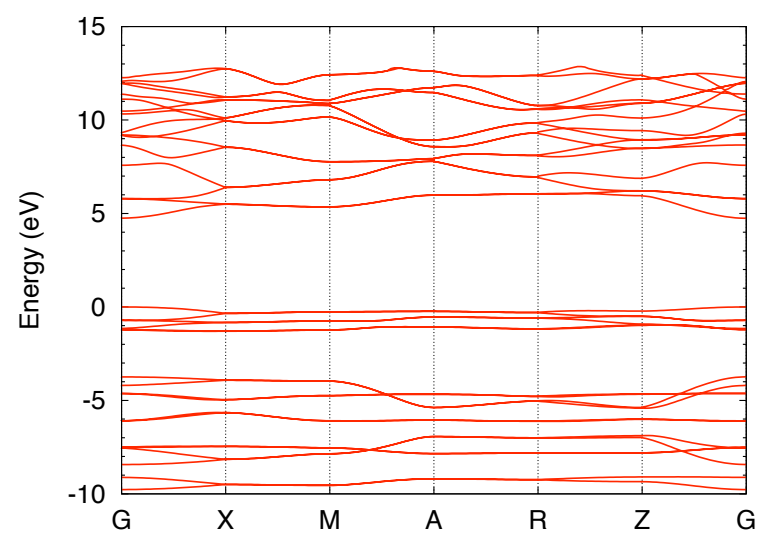

Fig. 1: Band structure of urea obtained by Local Density Approximation. The energy bands show small dispersion and a direct band gap of 4.74 is observed at the gamma point (compared to the experimental value $6.18 \mathrm{eV}$ [16]). A scissors operator of $1.46 \mathrm{eV}$ is used to correct the LDA band gap to experiment and shifts the results to the right. The symmetry points in reciprocal space, listed along the $\mathrm{x}$-axis are $\mathrm{G}=(000)$, $X=\left(0 \frac{1}{2} 0\right), M=\left(\frac{1}{2} \frac{1}{2} 0\right), A=\left(\frac{1}{2} \frac{1}{2} \frac{1}{2}\right), R=\left(0 \frac{1}{2} \frac{1}{2}\right)$ and $Z=\left(00 \frac{1}{2}\right)$.

lated elastic cross sections in the low energy regime. We have thus concluded that omitting DEA from our calculations will have no or limited effects on the results.

\section{Results and Discussion. -}

Band structure and energy loss function. The obtained LDA band structure is illustrated in fig. 1, by the dispersion of eigenstates along high symmetry directions for the urea crystal in reciprocal space. The dispersion is found to be in good agreement with results found in the literature. First principle calculations on urea have been presented earlier $[46,47]$. There are some minor disagreements between the band structure presented in fig. 1 . and those presented in ref. [46,47], however due to the integration over the energy loss function with respect to energy (see ref. [34] for details), the impact of small differences in the band structure on the calculated inelastic cross section is negligible.

A direct band gap of $4.74 \mathrm{eV}$ is found at the gamma point (6.18 eV experimentally [16]), other parameters calculated are the Fermi energy with respect to the bottom of the valence band $\left(E_{\mathrm{f}}=12.81 \mathrm{eV}\right)$ and the valence band width $\left(E_{\mathrm{v}}=9.71 \mathrm{eV}\right)$. The energy loss function for zero momentum transfer is to our knowledge a feature absent in the literature, we obtain it from a linear response analysis within the random phase approximation and it provides a detailed structure for the energetics of the crystal within low-energy regime.

The calculated energy loss function for urea, $\operatorname{Im}\left[-\epsilon(q=0, E)^{-1}\right]$ is presented in fig. 2. In the absence of experimental data for urea, we provide a calculated ELF for ice and compare it to experimental data (inset in fig. 22. First principle calculations of 


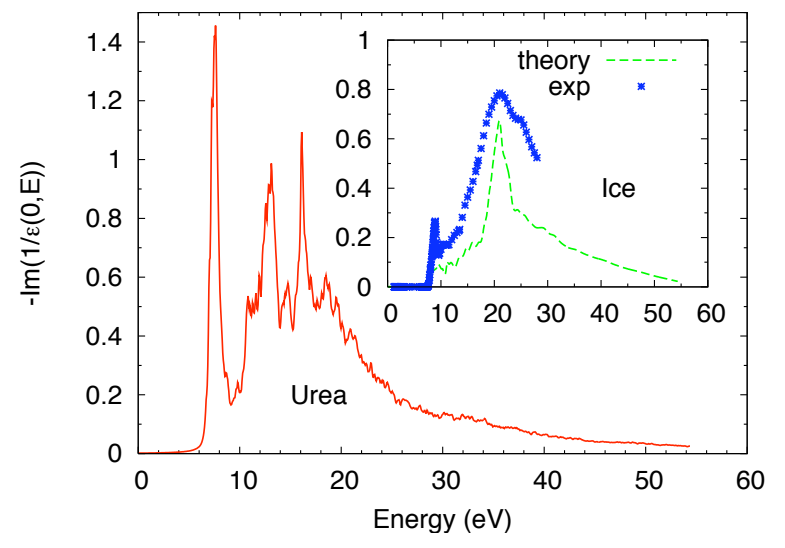

Fig. 2: Calculated energy loss function (ELF) of urea. The inset compares the calculated and experimental ELF [45] for ice. Due to the fewer number of van der Waals interactions in crystalline urea as compared to ice, we expect the calculations for urea to show better agreement with experiments than ice does. The first plasmon peak in urea is observed at $7.65 \mathrm{eV}$.

molecular crystals, where hydrogen bonds and van der Waals interactions are abundant, has been much debated in the past years without a consensus being reached. The debate regards which parametrization of the exchange and correlation contributions to the Coulomb potential is the most suitable, see for example reference [48] for a discussion on cohesive energies. Our LDA band gap and the characteristically flat dispersion of eigenstates is a good indication that the optical transition probabilities are being well described. Also, the close agreement between our calculations and experimental data for ice [45], as seen in inset in fig. 2, gives our urea results further support, as does a comparison with polystyrene [30] calculated based on a fit to optical data [49]. For higher energies $(>50 \mathrm{eV})$ the cross sections are generated within the Free Electron Gas approximation.

Inelastic electron cross section. The electron inelastic cross section for urea, together with those of water and diamond are presented in fig. 3. The water and diamond cross sections are presented as a comparison (taken from ref. [9]), both based on experimental energy loss functions. Being slightly denser than water, however with similar electronic properties, we expect the inelastic electron cross section to be higher than that for water (fig. 3). Comparing the inelastic electron cross section for urea to the estimated cross sections for other organic compounds, such as nucleic acids [29,30], we can also conclude that our calculations agree with earlier estimations of similar compounds. As compared to inelastic cross sections for free atoms, the cross sections for diamond (calculated using the approach employed in this work [34]) is around 50\% lower than that for free carbon atoms [50] (experimentally determined).

Ionization and electron dynamics. In contrast to earlier calculations of secondary electrons in water [9] and

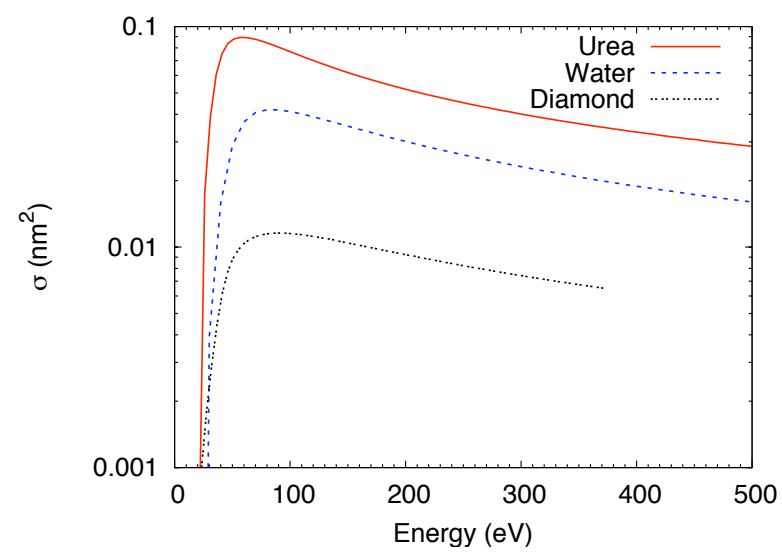

Fig. 3: Inelastic cross-section for electron scattering in urea. Diamond and water are plotted as a comparison, taken from ref. [9] and [34] respectively.

diamond [34], urea generates more secondary electrons from an initiating electron with the same energy. An electron with a kinetic energy of $278 \mathrm{eV}$ generates slightly above 12 secondary electrons in urea, whereas in water and diamond only 9 and 6 electrons are generated respectively. The number of electrons generated from a single impact electron for a certain compound is known to be related to the band gap of the material $[26,51]$ as $N_{\text {electron }} \sim E_{\text {init }} /\left(C E_{\text {gap }}\right)$, where $C$ is a compound specific constant. Fig. 4 illustrates how the average energy in the electron cloud drops down and equilibrates within a few femtoseconds. The figure shows the evolution of the energy distribution from an originating electron of $500 \mathrm{eV}$. The electron cascade in urea reaches energies below the ionization threshold after around 5 fs, which is twice as fast as seen in water. The electron energy loss is related to the density of the crystal, and urea is therefore expected to equilibrate faster than water. The main Auger decay lines in urea are at around $250 \mathrm{eV}$ (carbon), $400 \mathrm{eV}$ (nitrogen) and $500 \mathrm{eV}$ (oxygen). For these energies, the electron cascade generated by the Auger electron comprises of approximately 12 electrons (carbon), 18 electrons (nitrogen) and 24 electrons (oxygen).

In a small biomolecular sample exposed to an X-ray beam, the photoelectron is likely to escape the system, and it is the Auger electron only that will generate the secondary electron cascade. In such systems the sample will generate approximately as many electrons as a pure water sample, as water has slightly lower average Auger energy and a slightly lower density. This makes water a good model system for simulating X-ray damage as was done by Bergh et al. [2]. For larger systems however, the photoelectron does not escape, and using water as a model system underestimates the number of generated electrons by about $30 \%$.

In the energy regime that is investigated in the present work, we found that the total number of electrons generated after they have attenuated to subionization energies 


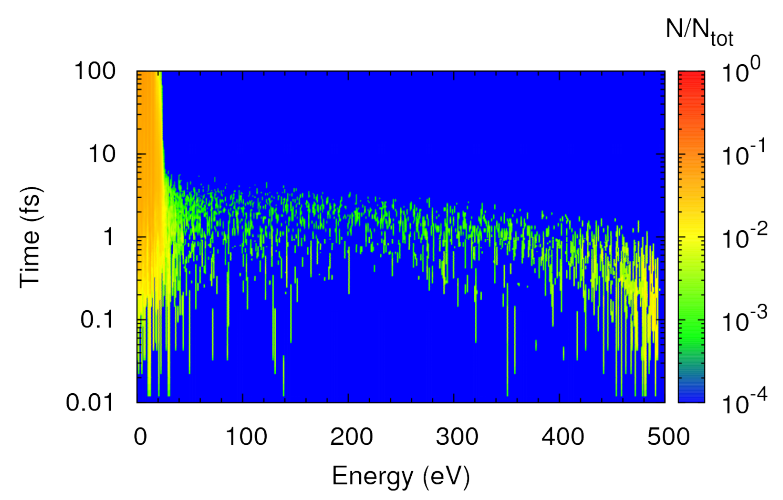

Fig. 4: Time evolution of the energy distribution in secondary electron cascade, generated from an initiating electron with a kinetic energy of $500 \mathrm{eV}$. The total number of electrons is normalized to 1 for every timestep (by dividing by the total number of electrons in the system). The figure shows an average over 100 simulations.

is linearly dependent on the energy of the initiating electron, as follows

$$
N_{\text {electron }}=\frac{E_{\text {init }}}{4.5 E_{\text {gap }}}
$$

Similar behaviour has been shown before for semiconductors $[10,51,52]$ as well as for diamond [26]. Damage due to ionization is related to the density of ionization events. The radius of gyration (i.e. the average distance of the electrons to the center of mass) of the generated electron cloud is not linear dependent on the initiating electron energy, but rather as a second order polynomial of the energy (fig. 5). This implies that the electron cloud from a low energy electron is much more dense, than that from a high energy electron, and therefore more damaging, despite the lower number of total electrons generated. The size of the ionizing electron cloud is quantified by the radius of gyration of the cloud. This relates to the energy of the initiating electron, which serves as a basis for understanding how the damage process depends with size in various crystals.

Since the cross sections are calculated for a neutral system, we do not explicitly create positive charges in the system and only account for the number of electrons lost. For a highly ionized system, the use of atomic cross sections will no longer be accurate, a plasma approximation is then needed [6]. This lapse in the model is likely to have an impact on the total number of electrons generated and remains to be studied. Furthermore, omitting the positive charged ions will lead to a larger electron cloud than expected, since we cannot account for shielding effects. However, for a system that is only fragmentary ionized, the impact of disregarding the positive charges should be negligible.

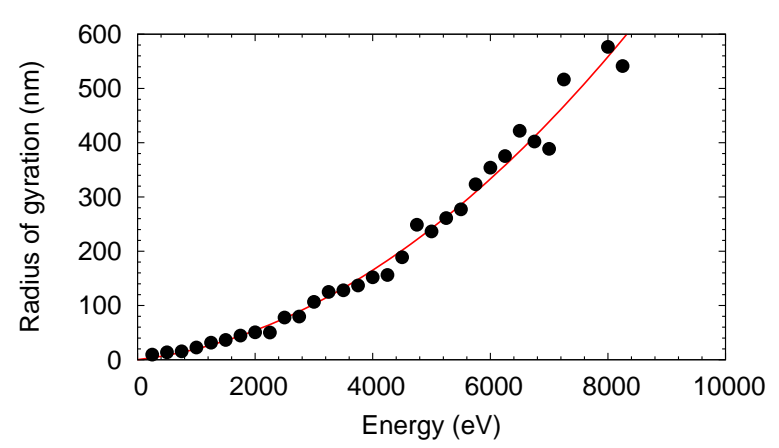

Fig. 5: Radius of gyration (average distance from center of mass) of the electron cloud as a function of the energy of the initiating electron, after 50 fs. Each dot represents the average over 50 simulations, the line is a fit to the dots, a second order polynomial: $y(x)=0.18+0.013 x+7.14 \times 10^{-6} x^{2}$.

Conclusion. - We have calculated the band structure and energy loss function for urea crystal from first principles. Employing a methodology presented and tested earlier, we have determined the inelastic electron cross section and estimated the number of secondary electrons generated from an incident electron at different energies. We are confident that the similarity of the ELF with experimental data and previous calculations on similar molecules implies our predictions are good models of reality. Our simulations show that urea generates $30 \%$ more electrons than water, from an initiating electron carrying the same energy. Such a difference is expected considering the density difference of urea and water. Urea bears more similarities with the biological matter (such as cells, viruses) in both elemental composition and density, making it a more suitable object of theoretical study than water or diamond, especially when addressing large systems. Furthermore we describe the size of the electron cloud generated from a single electron as a function of the electron energy, showing the spatial extent of the radiation damage due to secondary ionization. Secondary electron generation is an important effect for the damage caused on a biomolecular sample by an intense X-ray beam, and it is therefore necessary to understand these processes in order to predict the damage and aid the structural determination of single molecules or crystals.

$$
* * *
$$

The following organizations are acknowledged for their financial support: the Swedish Research Foundation (thanked by CO, EM, MG, FB, MK, JH, NT), Göran Gustafssons Stiftelse (thanked by $\mathrm{CO}$ and MK) and the DFG Cluster of Excellence: Munich-Centre for Advanced Photonics (thanked by CC, FGP and JH). Although not as cool as ice, or as shiny as diamond, the present work on 
urea has been endorsed by the following people, to whom we give thanks: Magnus Bergh, David van der Spoel, Richard London and Beata Ziaja.

\section{REFERENCES}

[1] Neutze R., Wouts R., van Der Spoel D., Weckert E. and HAJdu J., Nature, 406 (2000) 752.

[2] Bergh M., Tîmneanu N. and van der Spoel D., Phys. Rev. E, 70 (2004) 051904.

[3] Hau-Riege S. P., London R. A. and Szőke A., Phys. Rev. E , 69 (2004) 051906.

[4] Jurek Z., Faigel G. and Tegze M., Eur. Phys. J. D, 29 (2004) 217.

[5] Ziaja B., de Castro A., Weckert E. and Möller T., Eur. Phys. J. D, 40 (2006) 465.

[6] Bergh M., Tîmneanu N., Hau-Riege S. P. and Scott H. A., Phys. Rev. E , 77 (2008) 026404.

[7] Lennon M. A., Bell K. L., Gilbody H. B., Hughes J. G., Kingston A. E., Murray M. J. and Smith F. J., J. Phys. Chem. Ref. Data, 17 (1988) 1285.

[8] Günter F., Becker S., Schramm U., Eichner T., Fuchs M., Weingartner R., Habs D., Meyer-Tervehn J., Geissler M., Ferrario M., Serafini L., van Der Geer B., Backe H., Lauth W. and Reiche S., Appl. Phys. B , 86 (2007) 431.

[9] Tîmneanu N., Caleman C., Hajdu J. and van der Spoel D., Chem. Phys. , 299 (2004) 277.

[10] Ortiz C. and Caleman C., J. Phys. Chem. C. , 111 (2007) 17442.

[11] Van der Spoel D., Lindahl E., Hess B., Groenhof G., Mark A. E. and Berendsen H. J. C., J. Comp. Chem., 26 (2005) 1701.

[12] Pryor A. W. and Sanger P. L., Acta Cryst. A, 26 (1970) 543.

[13] Mullen D. and Hellner E., Acta Cryst. B , 34 (1978) 1624.

[14] Ortiz C., Eriksson O. and Klintenberg M., Comput. Mater. Sci. , (2008) in press.

[15] Gonze X., Beuken J., Caracas R., Detraux F., Fuchs M., Rignanese G., Sindic L., Verstraete M., rah G. Z., Jollet F., Torrent M., Roy A., Mikami M., Ghosez P., Raty J. and Allan D., Comput. Mater. Sci. , 25 (2002) 478.

[16] Donaldson W. and Tang C., Appl. Phys. Lett. , 44 (1984) 25.

[17] Bethe H., Ann. Phys., 5 (1930) 325.

[18] Lindhard J., K. Dan. Vidensk. Selsk. Mat. Fys. Medd., 28 (1954) 1.

[19] Penn D. R., Phys. Rev. B , 13 (1976) 674.

[20] Penn D. R., Phys. Rev. B , 35 (1987) 482.

[21] Tanuma S., Powell C. J. and Penn D. R., Surf. Interface Anal., 11 (1988) 577.

[22] Tanuma S., Powell C. J. and Penn D. R., Surf. Interface Anal. , 21 (1993) 165.

[23] Ashley J. C., J. Electron Spectrosc. Relat. Phenom, 46 (1988) 199.

[24] Ashley J. C., J. Electron Spectrosc. Relat. Phenom, 50 (1990) 323.

[25] Ashley J. C., J. Appl. Phys, 69 (1991) 674.
[26] Ziaja B., London R. A. and Hajdu J., J. Appl. Phys. 97 (2005) 064905.

[27] LaVerne J. A. and Pimblott S. M., Radiat. Res. , 141 (1995) 208.

[28] Tanuma S., Powell C. J. and Penn D. R., Surf. Interface Anal. , 20 (1993) 77.

[29] Tan Z., Xia Y., Zhao M. and Liu X., Radiat. Environ. Biophys. , 45 (2006) 135.

[30] Tan Z., Xia Y., LiU X., Zhao M., Ji Y., Li F. and Huang B., Radiat. Environ. Biophys. , 43 (2004) 173.

[31] Fung A. Y. C. and Zaider M., Radiat. Prot. Dosim. , 52 (1994) 111.

[32] BARIERI A. and VAN Hove M. A., private communications; phase shift package http://electron.lbl.gov/leedpack.

[33] Bransden B. H. and Joachain C. J., Physics of Atoms and Molecules (Longman, Essex) 1998.

[34] Ziaja B., van Der Spoel D., SzŐKe A. and Hajdu J., Phys. Rev. B , 64 (2001) 214104.

[35] Gabrysch M., Marklund E., Hajdu J., Twitchen D. J., Rudati J., Lindenberg A. M., Caleman C., Falcone R. W., Tschentscher T., Moffat K., Bucksbaum P. H., Als-Nielsen J., Nelson A. J., Siddons D. P., Emma P. J., Krejcik P., Schlarb H., Arthur J., Brennan S., Hastings J. and Isberg J., J. Appl. Phys., 103 (2008) 064909.

[36] Muroya Y., Meesungnoen J., Jay-Gerin J.-P., Filali-Mouhim A., Goulet T., sumura Y. K. and Mankhetkorn S., Can. J. Chem., 80 (2002) 1367.

[37] Silver M. and Sharma R., J. Comp. Phys. , 46 (1966) 692.

[38] Kalinowski J., Camaioni N., Marco P. D., Fattori V. and Martelli A., Appl. Phys. Lett., 72 (1998) 513.

[39] Chen J., Ma D., Liu Y. and Wang Y., J. Phys. D , 38 (2005) 3366.

[40] Johnson M. R., Parlinski K., Natkaniec I. and HudSON B., Chem. Phys., 291 (2003) 53.

[41] Boudaiffa B., Cloutier P., Hunting D., Huels M. and SANChE L., Science, 287 (2000) 1658.

[42] Huels M., Hahndorf I., Illenberger E. and Sanche L., J. Chem. Phys., 108 (1998) 1309.

[43] Breton S.-P., Michaud M., Jaggle C., Swiderek P. and Sanche L., J. Chem. Phys., 121 (2004) 11240.

[44] Lepage M., Michaud M. and Sanche L., J. Chem. Phys., 113 (2000) 3602.

[45] Warren S. G., Appl. Opt. , 23 (1984) 1206.

[46] Dovesi R., Causa' M., Orlando R. and Roetti C., J. Chem. Phys., 92 (1990) 7402.

[47] Lin Z., Wang Z., Chen C. and Lee M., J. Chem. Phys. , 118 (2003) 2349.

[48] Civalleri B., Doll K. and Zicovich-Wilson C. M., J. Phys. Chem. B. , 111 (2007) 26.

[49] Inagaki T., Arakawa E., Hamm R. N. and Williams M. W., Phys. Rev. B , 15 (1977) 3243.

[50] Brook E., Harrison M. F. A. and Smith A. C. H., J. Phys. B , 11 (1978) 3115.

[51] Klein C. A., J. Appl. Phys. , 39 (1968) 2029.

[52] Alig R. C., Bloom S. and Struck C. W., Phys. Rev. B , 22 (1980) 5565. 